\title{
Décompression du nerf alvéolaire inférieur par ostéotomie sagittale mandibulaire : un cas
}

\author{
Véronique Guillerminet ${ }^{1,{ }^{*}}$, Brice Chatelain ${ }^{1}$, Frédéric Denis ${ }^{1,2}$, Marie Pecheur ${ }^{1}$, Christophe Meyer ${ }^{1,3}$ \\ Service de chirurgie maxillo-faciale, de stomatologie et d'odontologie hospitalière, CHU de Besançon, France \\ Laboratoire de Neurosciences (EA-481)-UFC \\ Laboratoire I4S (EA INSERM 4268 - Pr B. Kastler) - UFC \\ veroguill@hotmail.com
}

(Reçu le 11 octobre 2014, accepté le 1 février 2015)

Mots clés :

complications postopératoires /

nerf alvéolaire inférieur /

lésion du nerf trijumeau /

traitement endodontique

Key words:

Postoperative complications /

mandibular nerve /

trigeminal nerve injury /

Endodontics
Résumé - Introduction : La compression du nerf alvéolaire inférieur (NAI) fait partie des complications possibles lors du traitement endodontique des troisièmes molaires mandibulaires par dépassement de matériel endodontique. Elle entraîne une atteinte sensitive dans le territoire du NAI correspondant dont la prise en charge est encore mal codifiée. Dans ce contexte, nous présentons une décompression chirurgicale du NAI par volet cortical latéral. Observation : Une patiente de 25 ans a consulté pour la prise en charge d'une anesthésie labio-mentonnière apparue 3 jours auparavant lors du traitement endodontique de la 38 et non résolutive suite à l'avulsion de cette dent. Le bilan radiologique montrait une fuite massive de pâte d'obturation dans le canal mandibulaire. Une décompression chirurgicale du NAI a été effectuée au piézotome au 7e jour sous anesthésie locorégionale. Discussion : La décompression chirurgicale du NAI doit être effectuée par un chirurgien expérimenté. Elle doit être réalisée rapidement (neurotoxicité des matériaux, inflammation, ischémie nerveuse). La technique doit être la moins traumatique possible pour permettre d'exposer le NAI sans le léser et préserver le volume osseux.

\begin{abstract}
Inferior alveolar nerve decompression by mandibular sagittal osteotomy. Case study. Introduction: The compression of the inferior alveolar nerve (IAN) is among the potential complications that may occur during endodontic treatment of the mandibular third molars due to a leak of the endodontic paste. It results in sensory loss in the corresponding territory of the IAN. Its treatment is not well defined. In this context, we present an IAN surgical decompression by lateral cortical window. Observation: A twentyfive-year-old patient was referred to us by her dentist on day three, to treat a numbness of the IAN that arose abruptly during endodontic treatment of the mandibular left third molar (38). The numbness remained, even following removal of the tooth. X-rays showed a significant leak of the endodontic paste into the mandibular canal. Surgical decompression of the IAN was performed with a piezotome on day seven under local anesthesia. Discussion: Surgical decompression of the IAN should be performed by an experienced surgeon. It must be performed quickly (neurotoxicity materials, inflammation, nerve ischemia), using a technique as atraumatic as possible to bring out the IAN with a minimum of additional damage and to preserve bone volume.
\end{abstract}

\section{Observation}

Une patiente de 25 ans a été adressée pour prise en charge d'une anesthésie labio-mentonnière gauche survenue brutalement trois jours auparavant au cours du traitement endodontique de la dent 38. À noter dans les antécédents de la patiente un déficit en protéines $C$ et $S$ traité par antivitamine $\mathrm{K}$ (AVK) (fluindione). L'examen clinique confirmait l'anesthésie labiomentonnière et révélait l'absence de 38, extraite en urgence par le praticien ayant réalisé le traitement endodontique. La radiographie rétro-alvéolaire montrait un dépassement important de matériaux d'obturation canalaire (OC) (association de paraformaldéhyde et d'eugénol) à l'apex de 38. La radiographie panoramique montrait un dépassement diffus de produit d'OC au fond de l'alvéole de 38 extraite, fusant massivement dans le canal mandibulaire (Fig. 1a). Le bilan biologique indiquait un INR à 4 . Après une attente de 4 jours permettant une normalisation de l'INR à 2,7, nous sommes intervenus au 7 e jour post-complication pour pro- 


\section{A S C L I N I Q U E}

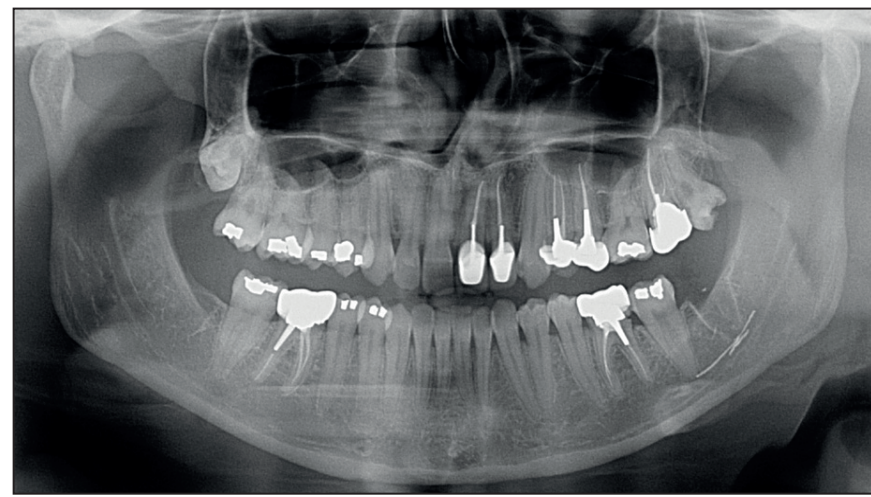

Fig. 1a. Orthopantomogramme préopératoire.

Fig 1a. Orthopantomogram preoperative.

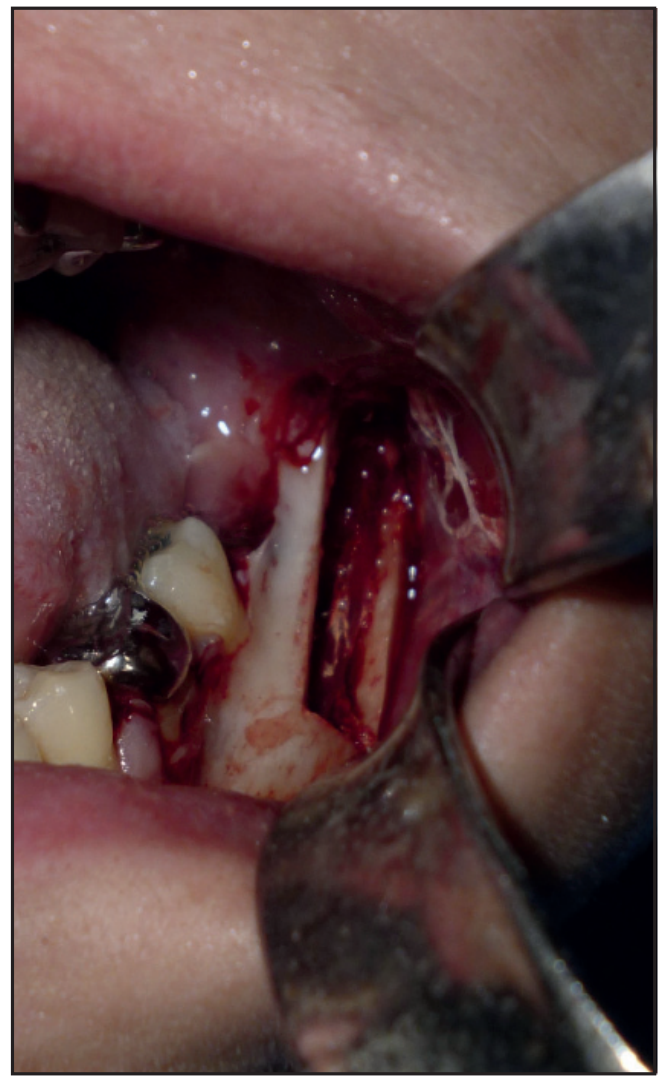

Fig. 1b. Ostéotomie sagittale : vue peropératoire.

Fig. 1b. Mandibular sagittal osteotomy: peroperative view.

céder, sous anesthésie locorégionale, à une décompression du NAI. Le canal mandibulaire a été ouvert sur toute sa longueur à l'aide d'un volet cortical latéral réalisé au piézotome (Fig. 1b). Sous contrôle visuel, nous avons procédé à l'exérèse du produit d'OC, au dégagement et au lavage au sérum physiologique du NAI. En fin d'intervention, le volet cortical a été replacé et maintenu par ostéosynthèse ( 2 plaques titane 2 trous ; 4 vis titane de 1,5 $\mathrm{mm}$ de diamètre et de $4 \mathrm{~mm}$ de

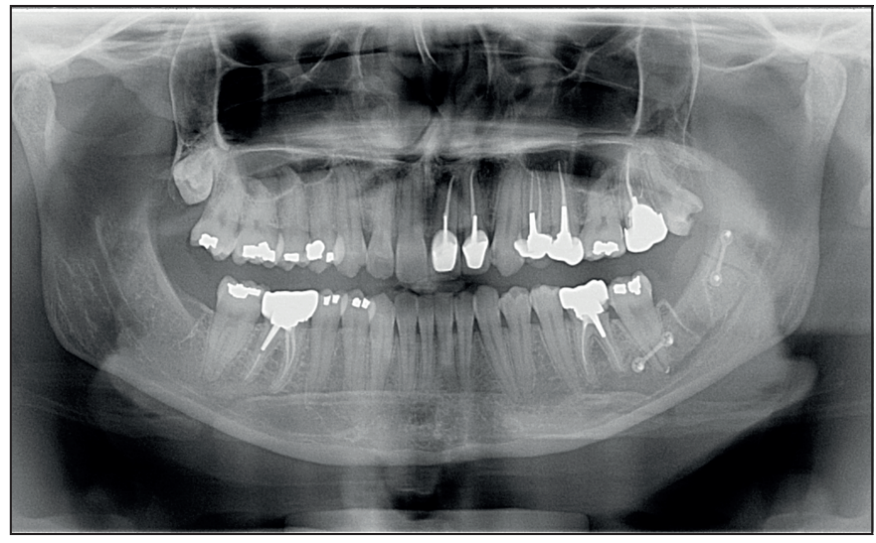

Fig. 1c. Orthopantomogramme postopératoire. Fig. 1c. Orthopantomogram postoperative view.

long ; Medartis ${ }^{\circledR}$, Bâle, Suisse, ) (Fig. 1c). Après l'intervention, notre patiente a été mise sous antibiotiques (amoxicillineacide clavulanique 3 grammes par jour), corticoïdes (prednisolone $40 \mathrm{mg}$ par jour), antiseptique (chlorhexidine 3 fois par jour) et vitamines B1, B6 (3 gélules par jour).

À six mois postopératoire, la patiente avait partiellement récupéré sa sensibilité labiomentonnière gauche mais il persistait des paresthésies labiales et des hyperesthésies douloureuses intermittentes. Afin d'améliorer sa qualité de vie, une prise en charge spécialisée de la douleur a été instaurée (chlorhydrate $\mathrm{d}^{\prime}$ oxycodone, Oxynorm ${ }^{\circledR}$ ).

\section{Discussion}

Une prévalence de 3 à $13 \%$ de lésions du NAI suite à un traitement endodontique est rapportée dans la littérature. Elle est de l'ordre de $5 \%$ sur les molaires mandibulaires avec une plus forte prévalence pour la $2 \mathrm{e}$ molaire mandibulaire. La proximité anatomique du NAI avec les apex des molaires mandibulaires constitue un facteur de risque évident [1]. Les conséquences pour le NAI d'un dépassement de pâte d'OC sont doubles : d'une part, la compression du nerf par le matériau et, d'autre part, les risques liés à la neurotoxicité du matériau. Les matériaux les plus neurotoxiques seraient ceux contenant du paraformaldéhyde ou ses analogues. En cas de lésion de l'épinerve, les fascicules nerveux seraient plus exposés à la toxicité des matériaux $[2,3]$. La prise en charge de cette complication comporte plusieurs axes. En premier lieu, il convient de prévenir cet incident par un protocole rigoureux lors d'un traitement canalaire : radiographie préopératoire, repérage de la longueur de travail (radiographie peropératoire et/ou localisateur d'apex), respect du péri-apex lors de l'instrumentation et de l'obturation canalaire. Dans ce cas, les « bourres pâtes » type lentulo ou pastinject sont à proscrire.

Compte tenu d'une composante inflammatoire liée au traumatisme physique et chimique du nerf, les corticoïdes peuvent 


\section{A S C L I N I Q U E}

être proposés en phase aiguë, même s'il n'existe pas de consensus ou de recommandations claires à ce sujet [4].

Les techniques chirurgicales de décompression sont multiples. La simple avulsion de la dent causale nous semble insuffisante du fait des mécanismes à la fois mécaniques et chimiques. Les décompressions par voies alvéolaires et/ou apicales sont, dans les cas de dépassement massif le long du canal mandibulaire, insuffisantes. De plus, elles exposent à un risque important de lésion iatrogène surajouté du fait de la visibilité très limitée que permettent ces voies d'abord.

La décompression et la toilette chirurgicale du nerf par ouverture large du canal mandibulaire réalisées par un praticien entraîné à la chirurgie orale sont pour nous la meilleure thérapeutique.

Dans ce contexte, la réalisation d'un volet cortical latéral présente l'intérêt, par rapport à une ouverture progressive du canal par décortication, de mettre en évidence le nerf alvéolaire inférieur avec un minimum de risque de lésions supplémentaires. En effet, la distance importante $(10 \mathrm{~mm}$ en moyenne) entre la corticale osseuse vestibulaire et le nerf alvéolaire inférieur en regard de la 3e molaire, rend l'accès difficile, par manque de visibilité et entraîne un délabrement osseux important. Le piézotome offre l'avantage d'une coupe fine, atraumatique et sans vibration parasite [5]. La précocité de cette décompression est un facteur de bon pronostic. En cas de symptomatologie douloureuse installée, le traitement devient celui des douleurs neuropathiques [6].

\section{Conflits d'intérêt : aucun}

\section{Références}

1. Alves FR, Coutinho MS, Gonçalves LS. Endodontic-related facial paresthesia: systematic review. J Can Dent Assoc 2014;80:e13.

2. Koseoglu BG, Tanrikulu S, Subay RK, Sencer S. Anesthesia following overfilling of a root canal sealer into the mandibular canal: a case report. Oral Surg Oral Med Oral Pathol Oral Radiol Endod 2006;101(6):803-806.

3. Pogrel MA. Damage to the inferior alveolar nerve as the result of root canal therapy. J Am Dent Assoc 2007; 138(1):65-69.

4. Chen N, Yang M, He L, Zhang D, Zhou M, Zhu C. Corticosteroids for preventing postherpetic neuralgia. Cochrane Database Syst Rev 2010;8(12):CD005582.

5. Goyal M, Marya K, Jhamb A, Chawla S, Sonoo PR, Singh $V$, et al. Comparative evaluation of surgical outcome after removal of impacted mandibular third molars using a Piezotome or a conventional handpiece: a prospective study. Br J Oral Maxillofac Surg 2012;50(6):556-561.

6. Scala R, Cucchi A, Cappellina L, Ghensi P. Cleaning and decompression of inferior alveolar canal to treat dysesthesia and paresthesia following endodontic treatment of a third molar. Indian J Dent Res 2014;25(3):413-415. 